\title{
AN ENERGY-BASED HYSTERESIS MODEL FOR MAGNETOSTRICTIVE TRANSDUCERS
}

\author{
F.T. Calkins \\ Department of Aerospace Engineering \\ and Engineering Mechanics \\ Iowa State University \\ Ames, IA 50011 \\ tcalkins@iastate.edu \\ R.C. Smith \\ Department of Mathematics \\ Iowa State University \\ Ames, IA 50011 \\ rsmith@iastate.edu \\ A.B. Flatau \\ Department of Aerospace Engineering \\ and Engineering Mechanics \\ Iowa State University \\ Ames, IA 50011 \\ abf@iastate.edu
}

\begin{abstract}
This paper addresses the modeling of hysteresis in magnetostrictive transducers. This is considered in the context of control applications which require an accurate characterization of the relation between input currents and strains output by the transducer. This relation typically exhibits significant nonlinearities and hysteresis due to inherent properties of magnetostrictive materials. The characterization considered here is based upon the Jiles-Atherton mean field model for ferromagnetic hysteresis in combination with a quadratic moment rotation model for magnetostriction. As demonstrated through comparison with experimental data, the magnetization model very adequately quantifies both major and minor loops under various operating conditions. The combined model can then be used to accurately characterize output strains at moderate drive levels. The advantages to this model lie in the small number (six) of required parameters and the flexibility it exhibits in a variety of operating conditions.
\end{abstract}




\section{Introduction}

This paper addresses the modeling of hysteresis in magnetostrictive transducers. The capabilities for actuation and sensing in such transducers are provided by the dual magnetostrictive effects in the core material: (i) the application of a magnetic field generates strains in the material and (ii) material stresses yield measurable magnetic effects. One core magnetostrictive material which has proven very effective at room temperatures and nominal operating conditions is Terfenol-D (see [1,2] for descriptions of the material and its capabilities). Due to the magnitude of the strains and forces generated by the material, Terfenol-D transducers have been employed as ultrasonic transducers, sonar projectors and provide the capability for controlling vibrations in heavy structures and industrial machinery.

Several properties inherent to magnetostrictive materials must be addressed when designing systems which employ them. The first concerns the hysteresis and nonlinear dynamics exhibited by the materials. This is due to inherent magnetic properties of the materials and is particularly pronounced at higher drive levels. It is also well documented that Terfenol-D performance is highly sensitive to operating conditions such as temperature, mechanical prestress, magnetic excitation (bias and AC amplitude), frequency and external load [3, 4, 5]. Several of these aspects (e.g., prestress and external loads) involve system aspects external to the core Terfenol-D material which makes the extrapolation of results from isolated laboratory samples to actual transducer design difficult and motivates consideration of the transducer as a whole.

Accurate modeling of transducer dynamics is necessary to take advantage of the full capabilities of the materials and to provide the ability for tailoring the performance of the transducers by modifying easily adjusted operating conditions. To attain these objectives, the model must accurately characterize both major (symmetric) and minor (nested and asymmetric) hysteresis loops as well as constitutive nonlinearities. The model must also incorporate the sensitivities with respect to operating conditions and be in a form amenable for eventual incorporation in models for underlying structural systems. Finally, the model must be suitable for controller design in the sense that it is efficient to implement and characterizes all dynamics which may be specified by the control law. For example, a model which characterizes major loops but not minor ones would be less useful in a feedback control law which cannot differentiate between the two.

The model we consider is obtained through the extension of the ferromagnetic mean field model of Jiles and Atherton [6, 7, 8, 9] to magnetostrictive transducers. This provides a characterization for the inherent hysteresis which is based upon the anhysteretic magnetization along with reversible and irreversible domain wall movements in the material. When coupled with nonlinear strain/magnetization relations, this yields a model which characterizes strain outputs in terms of input currents to the driving solenoid. Minor loops are incorporated through the enforcement of closure conditions.

With regard to design criteria, this model is currently constructed for a transducer with quasi-static input and fixed temperatures (these are commonly employed conditions for initial transducer characterization). The capability for having different prestresses and variable input magnitudes to the driving solenoid are included in the model and demonstrated through comparison with experimental data. The advantages of this approach lie in the accurate fits attainable in the considered regimes with a small number (six) of physical parameters 
to be identified through least squares techniques. This provides the method with significant flexibility and low computational overhead. The model is also in a form which can be extended to variable temperature and frequency regimes and can be incorporated in a large variety of structural models (e.g., see [10,11]). As a result, it shows great promise for use in transducer design for precision positioning and structural and structural acoustic controllers [12].

To place this modeling approach in perspective, it is useful to briefly summarize existing techniques for characterizing magnetostrictive transducers. For initial applications, linear field/magnetization relations were used to approximate the transducer dynamics [1, 13]. While this approach is reasonable at low drive levels, it is inaccurate at moderate to high input levels due to inherent hysteresis and material nonlinearities. In this latter regime, various phenomenological or empirical techniques, including Preisach models, have been employed to quantify the input/output relations $[14,15]$. Phenomenological approaches circumvent unmodeled or unknown physical mechanisms and have the advantage of generality. While some connections have been made between underlying physical processes and Preisach models [16], this genre of model typically provides less insight into physical dynamics than a model developed from physical principles. Furthermore, such empirical models generally require a large number of nonphysical parameters and are not easily adapted to changing operating conditions. This increases implementation time [17] and will limit flexibility if employed in a control law.

A typical magnetostrictive transducer is described in Section 2. This illustrates the system being modeled and indicates design issues which must be incorporated in the model. The energy-based model is discussed in Section 3 and the applicability of the model in a variety of experimental settings is presented in Section 4. These results illustrate the accuracy and flexibility of the model at fixed temperatures and low frequencies and indicate the extensions necessary for use in other regimes.

\section{Magnetostrictive Transducers}

The issues which must be addressed when developing a comprehensive model are illustrated in the context of the transducer depicted in Figure 1. As detailed in [14], this construction is typical for actuators currently employed in many structural applications; hence it provides a template for the development of models which will ultimately enhance design and performance. Details regarding the specific experimental setup used here are provided in Section 4.

From a design perspective, the transducer can be considered as the entire system which facilitates the utilization of the magnetostrictive core for applications. For modeling purposes, the key components are the magnetostrictive core, a DC magnetic circuit, a driving $\mathrm{AC}$ circuit and a prestress mechanism. The magnetostrictive material used in the transducer for the experiments reported in Section 4 was comprised of Terfenol-D, $\mathrm{Tb}_{0.3} \mathrm{Dy}_{0.7} \mathrm{Fe}_{1.9}$, while the driving AC magnetic field was generated by a surrounding wound wire solenoid. As illustrated by the experimental data plotted in Figure 2, the relationship between the applied field $H$ and resulting magnetization $M$ exhibits significant hysteresis while the relationship between the magnetization and strain $e$ is highly nonlinear. Moreover, the strains in an unbiased rod are always positive since the rotation of moments in response to an applied field always produce an increase in length. To attain bidirectional strains, a DC bias is provided by the enclosing 
cylindrical magnet (alternatively, a biasing DC current could be applied to the solenoid). Finally, the prestress bolt further aligns the orientation of magnetic moments and maintains the rod in a constant state of compression.

To fully utilize the transducer for structural applications and eventual controller design, it is necessary to characterize the relationship between the current $I$ applied to the solenoid, the resulting field $H$, the associated magnetization $M$ and finally, the generated strains $\epsilon$. A characterization based upon the Jiles-Atherton ferromagnetic hysteresis model is presented in the next section.

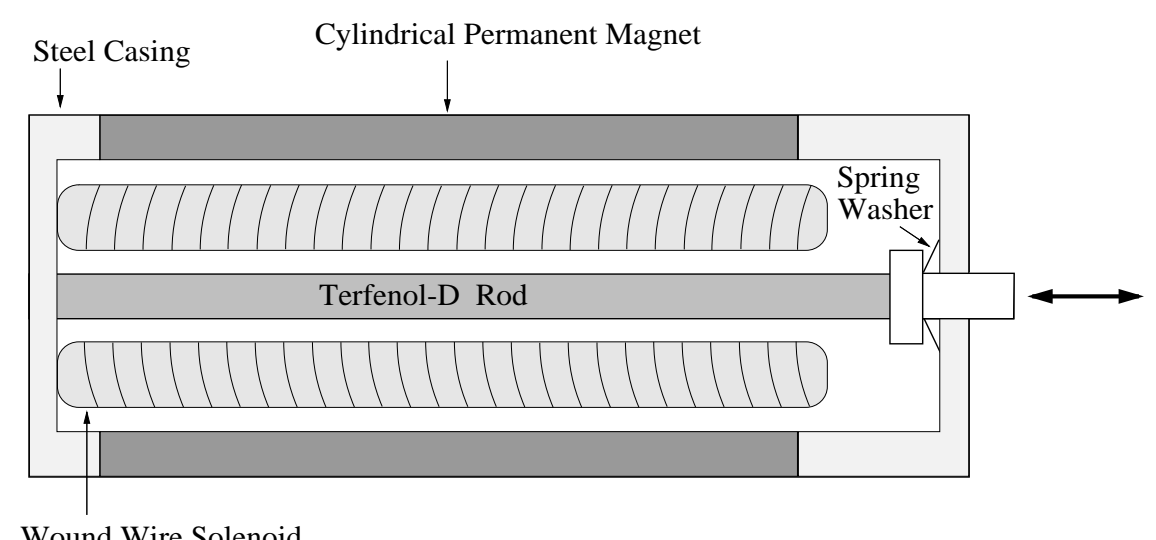

Figure 1. Cross section of a typical Terfenol-D magnetostrictive transducer.

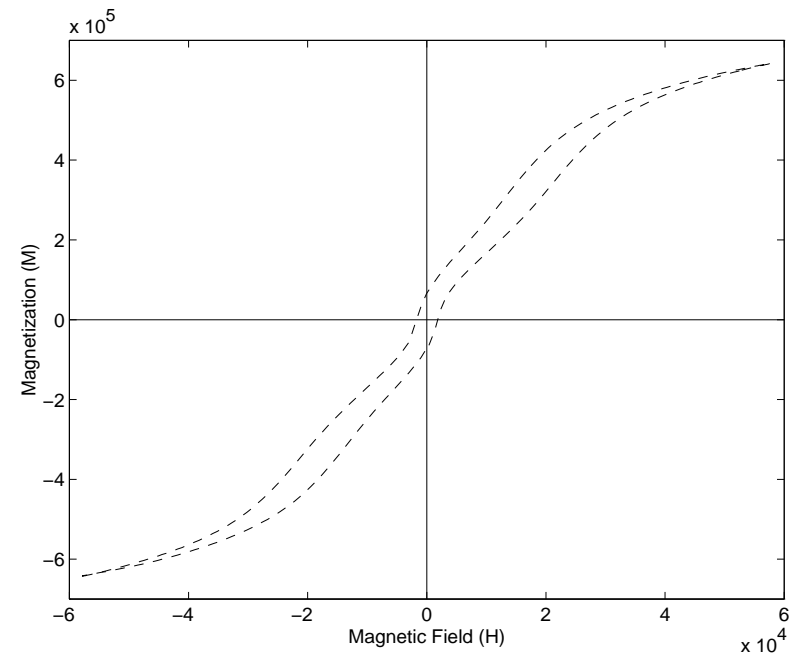

(a)

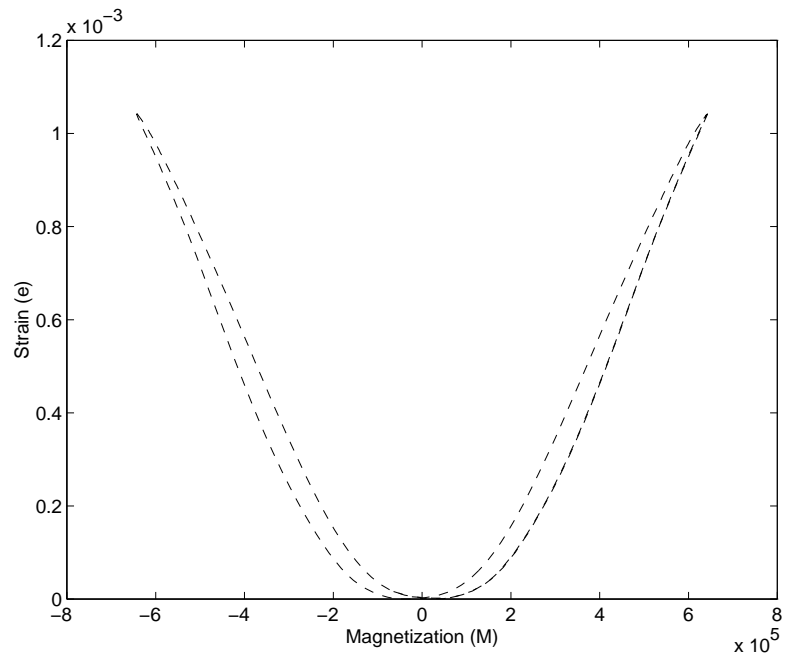

(b)

Figure 2. Relationship in experimental data between (a) the magnetic field $H$ and the magnetization $M$, and (b) the magnetization $M$ and the generated strains $e$. 


\section{Domain Wall Dynamics}

The transducer model described here is based upon the theory that magnetization in ferromagnetic materials is due to the realignment of magnetic moments within the material. Such materials exhibit the property that at temperatures below the Curie point, moments are highly aligned in regions termed domains (the reader is referred to $[6,18]$ for further discussion regarding the experimental verification of domain properties). The reorientation of moments can occur both in bulk within the domains or within transition regions, termed domain walls, between domains.

For a material which is defect free, the former mechanism leads to anhysteretic (hysteresis free) behavior which is conservative and hence reversible. Such a situation is idealized, however, since defects are unavoidable (e.g., carbides in steel) and in many cases, incorporated in the material to attain the desired stoichiometry (e.g., second-phase materials such as Dysprosium in Terfenol-D). These defects or inclusions provide pinning sites for the domain walls due to the reduction in energy which occurs when the domain wall intersects the site. For low magnetic field variations about some equilibrium value, the walls remain pinned and the magnetization is reversible. This motion becomes irreversible at higher field levels due to wall intersections with remote inclusions or pinning sites. Note that pinning effects lead to phenomena such as the Barkhausen discontinuities observed in experimental magnetization data $[6,18]$. The energy loss due to transition across pinning sites also provides the main mechanism for hysteresis in ferromagnetic materials.

\section{Magnetostriction}

The model presented here ultimately provides a relationship between the current $I$ input to the solenoid and the strain $e$ output by the transducer. As a first step, we characterize the magnetostriction which results at a given magnetization level. The magnetostriction $\lambda \equiv \frac{d \ell}{\ell}$ indicates the relative change in length of the material from the ordered but unaligned state to the state in which domains are aligned. While the magnetostriction does not quantify DC effects, the effects of domain order, or thermal effects, it does provide a measure of the strains generated in a Terfenol transducer.

As detailed in [6], consideration of the potential energy for the system yields

$$
\lambda=\frac{3}{2} \frac{\lambda_{s}}{M_{s}^{2}} M^{2}
$$

as a first approximation to the relationship between the magnetization and magnetostriction. Here $M_{s}$ and $\lambda_{s}$ respectively denote the saturation magnetization and saturation magnetostriction. For an isolated Terfenol-D sample, $M_{s}$ represents the magnetization required to rotate all moments and has been observed to have the approximate value $M_{s} \approx 7.9 \times 10^{5} \mathrm{~A} / \mathrm{m}[19]$. This parameter has a similar interpretation in the full transducer model but will be shown in the examples of the next section to have the slightly smaller value of $M_{s}=7.65 \times 10^{5} \mathrm{~A} / \mathrm{m}$. This illustrates the necessity of estimating such parameters for the specific transducer under consideration. The value of $\lambda_{s}$ depends upon the initial orientation of moments and hence upon the applied prestress. In the absence of applied stresses and under the assumption of a cubic anisotropy model, $\lambda_{s}$ can be defined in terms of the independent saturation magnetostrictions $\lambda_{100}$ and $\lambda_{111}$ in the $\langle 100\rangle$ and $\langle 111\rangle$ directions, respectively. As detailed in 
[6], under the assumption that the material contains a large number of domains and has no preferred grain orientation, averaging of domain effects yields the expression

$$
\lambda_{s}=\frac{2}{5} \lambda_{100}+\frac{3}{5} \lambda_{111}
$$

for the total saturation magnetostriction (typical saturation values for Terfenol are $\lambda_{100}=$ $90 \times 10^{-6}$ and $\left.\lambda_{111}=1600 \times 10^{-6}\right)$. As will be noted in the examples of the next section, this saturation value is highly dependent upon the operating conditions (e.g., applied prestress) and the parameter $\lambda_{s}$ must be estimated through least squares techniques for the specific conditions under consideration.

For the operating conditions under consideration, the quadratic expression (1) adequately models the relationship between the magnetization and strain at low to moderate drive levels. For higher drive levels and frequencies along with variable temperature and stress conditions, however, it must be extended to include higher-order mechanisms (e.g., the sensitivity of the system with regard to changing stress is an important and well documented phenomenon [3, $4,5]$ ). This can be accomplished through the incorporation of stress dependence in $\lambda_{s}$ and the use of higher-order magnetostrictive models as discussed in [20]. Alternatively, higher-order effects and magnetostrictive hysteresis can be incorporated through an energy formulation as detailed in [9]. Finally, the effects of magnetomechanical coupling and mechanical resonances must be incorporated in various operating regimes. Hence this component of the transducer model should be extended as dictated by operating conditions.

We next turn to the characterization of the magnetization $M$ in terms of the input current $I$. To accomplish this, it is necessary to quantify the effective field $H_{\text {eff }}$ associated with the magnetic moments in the core material, the anhysteretic magnetization $M_{a n}$, the reversible magnetization $M_{\text {rev }}$ and the irreversible magnetization $M_{i r r}$.

\section{Effective Magnetic Field}

In general, the effective field is dependent upon the magnetic field generated by the solenoid, magnetic domain interactions, crystal and stress anisotropies, and temperature. For this model, we are considering the case of fixed temperature and compressive prestresses in excess of $0.8 \mathrm{ksi}$. It is noted in the computations on pages 126 and 410 of [6] that for polychrystaline Terfenol, a compressive stress of $\bar{\sigma}=6.25 \mathrm{MPa}$ or $899 \mathrm{psi}$ is required to align moments perpendicular to the stress under the assumptions that $\lambda_{s}=1067 \times 10^{-6}$ and the crystal anisotropy constant is $K_{1}=-2 \times 10^{4} \mathrm{~J} / \mathrm{m}^{3}$. While this computed value of $\bar{\sigma}$ is highly dependent upon temperature and operating conditions, it indicates that stress anisotropies will start to dominate crystal anisotropies by $1 \mathrm{ksi}$ with the effect magnified at higher prestresses. This motivates the use of a model which neglects crystal anisotropies when operating in high stress regimes.

Under the assumption of fixed temperature and sufficiently large prestresses, the effective magnetic field is modeled by

$$
H_{e f f}=H+\alpha M+H_{\sigma}
$$

where $H=n I$ is the field generated by a solenoid with $n$ turns per unit length, $\alpha M$ quantifies the field due to magnetic interactions between moments, and $H_{\sigma}$ is the field due to magnetoelastic domain interactions. The parameter $\alpha$ quantifies the amount of domain interaction 
and must be identified for a given system. The field component due to the applied stresses can be quantified through thermodynamic laws to obtain

$$
H_{\sigma}=\frac{3}{2} \frac{\sigma}{\mu_{0}}\left(\frac{\partial \lambda}{\partial M}\right)_{\sigma, T}
$$

(see $[9,20]$ for details). Here $\mu_{0}$ is the free space permeability, and the subscript $T$ denotes constant temperature in degrees Kelvin. Note that with the approximation (1) for $\lambda$, the effective field can be expressed as

$$
H_{e f f}=H+\widetilde{\alpha} M
$$

where $\tilde{\alpha} \equiv \alpha+\frac{9}{2} \frac{\lambda_{s} \sigma}{\mu_{0} M_{s}^{2}}$.

\section{Anhysteretic Magnetization}

The anhysteretic magnetization is computed through consideration of the thermodynamic properties of the magnetostrictive material. Under the assumption of constant domain density $N$, Boltzmann statistics can be employed to yield the expression

$$
M_{a n}=M_{s} \mathcal{L}\left(H_{e f f} / a\right)
$$

where $\mathcal{L}(z) \equiv \operatorname{coth}(z)-1 / z$ is the Langevin function. The constant $a$ is given by $a=\frac{N k_{B} T}{\mu_{0} M_{s}}$ where $k_{B}$ is Boltzmann's constant and $k_{B} T$ represents the Boltzmann thermal energy. We point out that a cannot directly be computed for a transducer due to the fact that $N$ is unknown. Hence it is treated as a parameter to be identified for the system. We also note that this expression for $M_{a n}$ is valid only for operating conditions under which $H_{e f f}$ is valid. For example, if prestresses are sufficiently small so that crystal anisotropies are significant, the expression must be modified to incorporate the differing anisotropy energies in the different directions. One approach to modeling the effects of anisotropy is given in [21].

\section{Irreversible, Reversible and Total Magnetization}

The anhysteretic magnetization incorporates the effects of moment rotation within domains but does not account for domain wall dynamics. As noted previously, the consideration of domain wall energy yields additional reversible and irreversible components to the magnetization. The consideration of energy dissipation due to pinning and unpinning of domain walls at inclusions yields the expression

$$
\frac{d M_{i r r}}{d H}=\frac{M_{a n}-M_{i r r}}{k \delta-\tilde{\alpha}\left(M_{a n}-M_{i r r}\right) \frac{d M_{i r r}}{d M}}
$$

for the differential susceptibility of the irreversible magnetization curve $[7,20]$. The constant $k=\frac{n\left\langle\varepsilon_{\pi}\right\rangle}{2 m \mu_{0}(1-c)}$, where $n$ is the average density of pinning sites, $\left\langle\varepsilon_{\pi}\right\rangle$ is the average energy for $180^{\circ}$ walls, $c$ is a reversibility coefficient, and $m$ is the magnetic moment of a typical domain, provides a measure for the average energy required to break a pinning site. The parameter $\delta$ is defined to have the value +1 when $\frac{d H}{d t}>0$ and -1 when $\frac{d H}{d t}<0$ to guarantee that pinning always opposes changes in magnetization. In applications, $\delta$ can be directly 
determined from the magnetic field data while $k$ is identified for the specific transducer and operating conditions.

The reversible magnetization quantifies the degree to which domain walls bulge before attaining the energy necessary to break the pinning sites. As derived in [7], to first approximation, the reversible magnetization is given by

$$
M_{r e v}=c\left(M_{a n}-M_{i r r}\right) .
$$

The reversibility coefficient $c$ can be estimated from the ratio of the initial and anhysteretic differential susceptibilities [8] or through a least squares fit to data. Properties of all the model parameters are summarized in Table 1.

The total magnetization is then given by

$$
M=M_{r \epsilon \imath}+M_{i r r}
$$

with $M_{i r r}$ and $M_{r e v}$ defined by (3) and (4) and the anhysteretic magnetization given by (2). The full time-dependent model leading from input currents to output magnetization is summarized in Algorithm 1. When combined with (1), this provides a characterization of the output strains in terms of the current $I$ input to the solenoid. Note that this model is valid for fixed temperature and quasi-static operating conditions. The extension to more general operating conditions will involve the previously mentioned modifications to the effective field.

$$
\begin{array}{ll}
\text { (i) } & H(t)=n I(t) \\
\text { (ii) } & H_{\text {eff }}(t)=H(t)+\alpha M(t)+H_{\sigma}(t) \\
\text { (iii) } & M_{a n}(t)=M_{s}\left[\operatorname{coth}\left(\frac{H_{e f f}(t)}{a}\right)-\left(\frac{a}{H_{e f f}(t)}\right)\right] \\
\text { (iv) } & \frac{d M_{i r r}}{d H}(t)=n \frac{d I}{d t} \cdot \frac{M_{a n}(t)-M_{i r r}(t)}{k \delta-\widetilde{\alpha}\left[M_{a n}(t)-M_{i r r}(t)\right] \frac{d M_{i r r}}{d M}} \\
\text { (v) } & M_{r e v}(t)=c\left[M_{a n}(t)-M_{i r r}(t)\right] \\
\text { (vi) } & M(t)=M_{r e v}(t)+M_{i r r}(t)
\end{array}
$$

Algorithm 1. Time-dependent model quantifying the output magnetization $M(t)$ in terms of the input current $I(t)$. The parameter $\widetilde{\alpha}$ is given by $\tilde{\alpha}=\alpha+\frac{9}{2} \frac{\lambda_{s} \sigma_{0}}{\mu_{0} M_{s}^{2}}$ where $\sigma_{0}$ is the applied prestress. 


\begin{tabular}{|c|c|c|}
\hline Parameter & Physical Property & Effects on Model \\
\hline$\alpha$ & $\begin{array}{l}\text { Quantifies domain } \\
\text { interactions }\end{array}$ & $\begin{array}{l}\text { Increased values lead to steeper slopes } \\
\text { for anhysteretic and magnetization } \\
\text { curves. }\end{array}$ \\
\hline$a$ & $\begin{array}{l}\text { Shape parameter } \\
\text { for } M_{a n}\end{array}$ & Increased value decreases slope of $M_{a n}$. \\
\hline$k$ & $\begin{array}{l}\text { Average energy } \\
\text { required to break } \\
\text { pinning sites }\end{array}$ & $\begin{array}{l}\text { Increased value produces wider hystere- } \\
\text { sis curve and narrower minor loop. }\end{array}$ \\
\hline$c$ & $\begin{array}{l}\text { Reversibility coef- } \\
\text { ficient }\end{array}$ & $\begin{array}{l}\text { Decrease in value leads to wider hystere- } \\
\text { sis curve. }\end{array}$ \\
\hline$M_{s}$ & $\begin{array}{l}\text { Saturation } \\
\text { magnetization }\end{array}$ & $\begin{array}{l}\text { Increase leads to large saturation value } \\
\text { for magnetization. }\end{array}$ \\
\hline$\lambda_{s}$ & $\begin{array}{l}\text { Saturation } \\
\text { magnetostriction }\end{array}$ & $\begin{array}{l}\text { Increase leads to large saturation value } \\
\text { for magnetostriction. }\end{array}$ \\
\hline
\end{tabular}

Table 1. Physical properties and effects of model parameters $\alpha, a, k, c, M_{s}, \lambda_{s}$. The parameter $\tilde{\alpha}$ is then given by $\tilde{\alpha}=\alpha+\frac{9}{2} \frac{\lambda_{s} \sigma_{0}}{\mu_{0} M_{s}^{2}}$ where $\sigma_{0}$ is the applied prestress.

\section{Asymmetric Minor Loops}

The final aspect which we consider here concerns the modification of the model to incorporate minor (asymmetric) loops. Such loops occur when the sign of $\frac{d H}{d t}$ is reversed for a trajectory lying within the interior of the major loop. To preserve order in the sense that forward paths do not intersect, it is necessary that minor loops close. The model (5) can be employed for the first half of the minor loop but does not ensure closure. This property is incorporated in the model through the consideration of a working volume and volume fraction for either the magnetization or the reversible and irreversible components.

To illustrate the first case, we let $t_{0}, t_{1}$ and $t_{2}$ respectively denote the times when the minor loop starts, when it turns due to a change in the sign of $\frac{d H}{d t}$, and when it closes (see Figure 3 ). The corresponding values of the magnetic field and magnetization are $H\left(t_{0}\right), H\left(t_{1}\right), H\left(t_{2}\right)$ and $M\left(t_{0}\right), M\left(t_{1}\right), M\left(t_{2}\right)$. Note that in order to guarantee closure of the minor loop, it is necessary to require that $H\left(t_{0}\right)=H\left(t_{2}\right)$ and $M\left(t_{0}\right)=M\left(t_{2}\right)$. Direct integration of (5) yields

$$
M_{2}=M\left(t_{1}\right)+\int_{t_{1}}^{t_{2}} \frac{d M}{d s} d s
$$

which in general will not be equal to $M\left(t_{0}\right)$. To attain closure, we define

$$
M(t)=M\left(t_{1}\right)-\frac{M\left(t_{1}\right)-M\left(t_{0}\right)}{M_{2}-M\left(t_{1}\right)} \int_{t_{1}}^{t} \frac{d M}{d s} d s
$$

for $t \in\left[t_{1}, t_{2}\right]$. The magnetization values $M\left(t_{0}\right), M\left(t_{1}\right), M_{2}$ and $\frac{d M}{d s}$ are computed using (5). Through the inclusion of this volume fraction

$$
v_{m}=\frac{M\left(t_{1}\right)-M\left(t_{0}\right)}{M_{2}-M\left(t_{1}\right)},
$$


the magnetization is forced to satisfy the closure property $M\left(t_{2}\right)=M\left(t_{0}\right)$. A similar formulation of volume fractions for the component reversible and irreversible magnetizations is given in [22] while extensions of the model to accommodate more complex anhysteretic effects can be found in [23].

The viability of the model with minor loops closed via (6) is illustrated in the next section. We note that for the operating conditions targeted in this paper, the model accurately characterizes the transducer response including both major loops and nested minor loops.

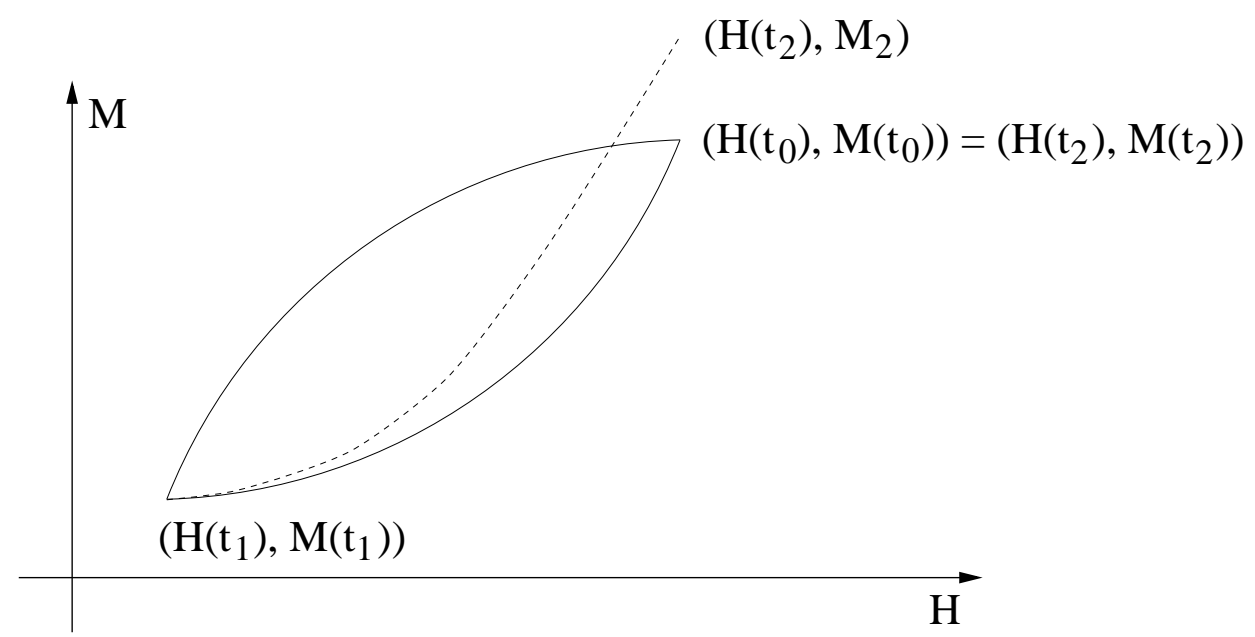

Figure 3. Closure requirements for minor loops.

\section{Model Fits to Experimental Data}

The model fits to experimental transducer data using the relations summarized in Section 3 are presented here. Following a description of the experimental transducer, two cases are considered. The first illustrates the performance of the magnetization and magnetostriction models under various drive levels with a $1.3 \mathrm{ksi}$ prestress applied to the Terfenol rod. Included in these results are model fits to data which contains minor loops. The second case illustrates the performance of the model for a prestress of $1.0 \mathrm{ksi}$. As discussed in the last section, the stress-dominated anisotropy model for the magnetization is valid for both cases. Taken in concert, these examples illustrate the accuracy and flexibility of the magnetization model for a range of drive levels, magnetic biases and prestresses for quasi-static operating conditions at fixed temperature. The quadratic magnetostriction model is also accurate at low to moderate drive levels but must be extended to incorporate the hysteresis and saturation present at high drive regimes.

\section{Experimental Transducer}

The experimental data reported here was collected from a broadband Terfenol-D transducer developed at Iowa State University. The nominal resonance range was designed for 
structural applications (1-10 kHz). Furthermore, the transducer was designed to produce an output free from spurious resonances and to permit adjustable prestress and magnetic bias.

The Terfenol-D $\left(\mathrm{Tb}_{0.3} \mathrm{Dy}_{0.7} \mathrm{Fe}_{1.9}\right)$ rod employed in the transducer had a length of $115 \mathrm{~mm}$ and a $12.7 \mathrm{~mm}$ diameter. The rod was placed inside two coils consisting of an inner single layer 110-turn pickup coil and a multi-layer 800-turn drive coil. A current control amplifier (Techron 7780) provided the input to the drive coil to produce an applied AC magnetic field and DC bias as necessary. The reference signal to this amplifier was provided by a Tektronix spectrum analyzer and the applied magnetic field $H$ generated by the drive coil had a frequency of $0.7 \mathrm{~Hz}$ and magnitude up to $5.6 \mathrm{kA} / \mathrm{m}(700 \mathrm{Oe})$ per ampere. The pickup coil was used to measure the induced voltage from which the time rate change of the magnetic induction $B$ was computed using the Faraday-Lenz law.

A cylindrical permanent magnet surrounding the coils provided the capability for generating additional DC bias if necessary. This permanent magnet was constructed of Alnico $\mathrm{V}$ and was slit to reduce eddy current losses. Note that for the experiments reported here, biases generated in this manner were unnecessary and the reported data is unbiased (i.e., the permanent magnet was demagnetized). Finally, mechanical prestresses to the rod were generated by a variable prestress bolt at one end of the transducer and Belleville washers fitted at the opposite end of the rod.

The measurable output from the transducer included the current and voltage in the drive coil, the voltage induced in the pickup coil, and the mechanical output. To quantify the mechanical output, a Lucas LVM-10 linear variable differential transformer based upon changing reluctance was used to measure the displacement of the transformer output interface connection. Temperature was maintained within $5^{\circ} \mathrm{C}$ of the ambient temperature $\left(23^{\circ} \mathrm{C}\right)$ by monitoring two thermocouples attached to the Terfenol-D sample.

\section{Parameter Estimation}

The use of the magnetization and magnetostriction models to characterize transducer dynamics requires the estimation of the parameters $\widetilde{\alpha}, a, k, c, M_{s}$ and $\lambda_{s}$ summarized in Table 1 . The parameters $\tilde{\alpha}, a, k$ and $c$ are in essence averages which arise when extending physics at a microscopic level to the macroscopic scale necessary for control implementation. Hence, while they have physical interpretations and tendencies, they must be estimated for individual transducers. The parameters $M_{s}$ and $\lambda_{s}$ are macroscopic and have published values for Terfenol under various operating conditions. Sufficient variation occurs in the values, however, that we also estimated them for the individual transducer.

The full set of parameters was estimated through a least square fit with experimental data from the previously described transducer. The optimization was performed in two steps. In the first, the values of $q=\left(\tilde{\alpha}, a, c, k, M_{s}\right)$ were estimated through minimization of the functional

$$
J(q)=\sum_{i=1}^{s}\left|M\left(t_{i} ; q\right)-z_{i}\right|^{2}
$$

where $z_{i}$ denotes the experimentally measured value of the Terfenol magnetization at time $t_{i}$. The modeled magnetization at time $t_{i}$ for parameter values $q$ is denoted by $M\left(t_{i} ; q\right)$ (see (5) or (vi) of Algorithm 1). The functional (7) was minimized using a constrained optimization algorithm based upon sequential quadratic programming (SQP) updates. 
With the estimated values of $\tilde{\alpha}, a, c, k$ and $M_{s}$, the model fits to the experimental magnetization curves can be obtained. The second step concerns the estimation of $\lambda_{s}$ to attain reasonable fits in the magnetostriction model (1). This was accomplished through a least squares fit with displacement or strain data from the transducer.

Initial magnetization parameters were estimated using this technique for the transducer with an applied prestress of $1.3 \mathrm{ksi}$. The resulting values are summarized in Table 2 while model fits are illustrated in Figure 4. From strain data, the saturation magnetostriction constant for this case was determined to be $\lambda_{s}=1003 \times 10^{-6}$ for high drive levels and $\lambda_{s}=1221 \times 10^{-6}$ at low drive levels (the difference in values is further discussed in the next section).

To ascertain the robustness of the model with respect to applied prestresses, we then considered the estimation of parameters and performance of the model with a prestress of $1.0 \mathrm{ksi}$. For this case, we fixed the parameters a, c, $M_{s}$ which have the least theoretical dependence upon prestress and estimated the parameters $k, \widetilde{\alpha}, \lambda_{s}$ through a least squares fit to the data. The estimated magnetization parameters are again summarized in Table 2 while the saturation magnetostriction was found to be $\lambda_{s}=995 \times 10^{-6}$ at high drive levels.

A comparison of the estimated values of $k$ indicates significant changes due to the effects of stress on the pinning energy at magnetic inclusions. The change in the saturation magnetostriction $\lambda_{s}$ is due to stress-induced changes in the initial domain configuration. The stress dependence in $\tilde{\alpha}=\alpha+\frac{9}{2} \frac{\lambda_{s} \sigma_{0}}{\mu_{0} M_{s}^{2}}$ is primarily due to magnetomechanical stress anisotropies which are quantified by the term $\frac{9}{2} \frac{\lambda_{s} \sigma_{0}}{\mu_{0} M_{s}^{2}}$. Note that for the compressive prestress $\sigma_{0}=-1 k s i \approx-6.9 \mathrm{MPa}$ and the estimated values for $\lambda_{s}, \widetilde{\alpha}, M_{s}$, the magnetic coupling parameter $\alpha$ has the computed value $\alpha=0.032$ while it has the value $\alpha=0.035$ for $\sigma_{0}=-1.3 k s i$. This small variation in the values of $\alpha$ (less than $9 \%$ ) illustrates the consistency of the model with regard to nearly constant applied stresses. Moreover, it indicates that one has the capability for identifying and fixing the parameter $\alpha$ and incorporating subsequent stress effects through the component $\frac{9}{2} \frac{\lambda_{s} \sigma_{0}}{\mu_{0} M_{s}^{2}}$. The use of this strategy has been substantiated by the highly accurate model fits obtained with fixed $\alpha$.

\begin{tabular}{l|l|l}
\hline & $\sigma_{0}=1.3 k s i$ & $\sigma_{0}=1.0 k s i$ \\
\hline$M_{s}(A / m)$ & $7.65 \times 10^{5}$ & $7.65 \times 10^{5}$ \\
$a(A / m)$ & 7012 & 7012 \\
$c$ & 0.18 & 0.18 \\
$k(A / m)$ & 3942 & 3283 \\
$\tilde{\alpha}$ & -0.02 & -0.01 \\
\hline
\end{tabular}

Table 2. Estimated magnetization parameters for the transducer with prestresses of $1.3 \mathrm{ksi}$ and $1.0 \mathrm{ksi}$. 


\section{Magnetization Model}

We consider first the performance of the quasi-static magnetization model summarized in Algorithm 1 under a variety of operating conditions. The model is formulated to be flexible with regard to various drive levels and prestresses and it was within this regime that the performance was tested. Data was collected at multiple drive levels with prestresses of $1.0 \mathrm{ksi}$ and $1.3 \mathrm{ksi}$ applied to the Terfenol rod. As detailed in [3], prestresses within this range yield nearly optimal magnetomechanical coupling and strain coefficients for the specific transducer. Parameters for the magnetization model were estimated through the previously described least squares techniques and used to obtain model responses under the various conditions. In each case, the measured applied field $H$ was used as input to the model.

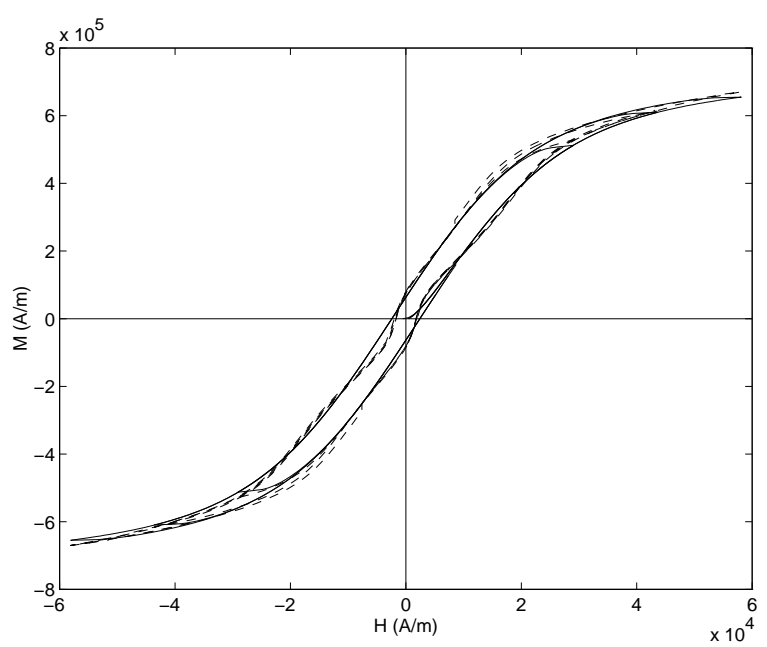

(a)

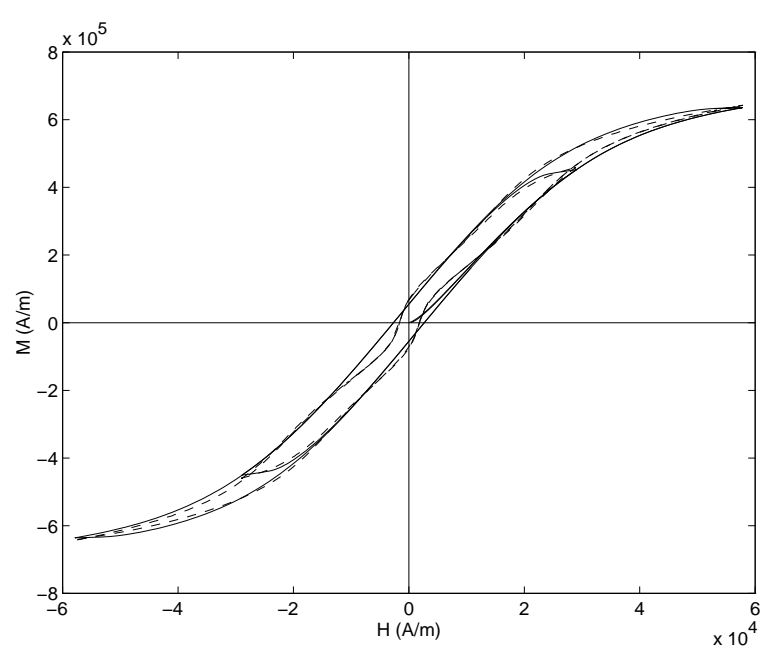

(c)

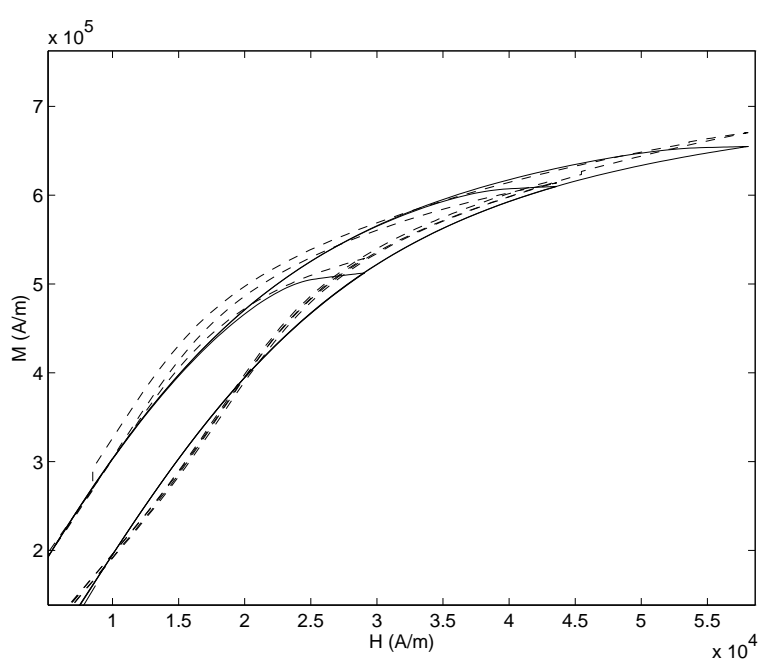

(b)

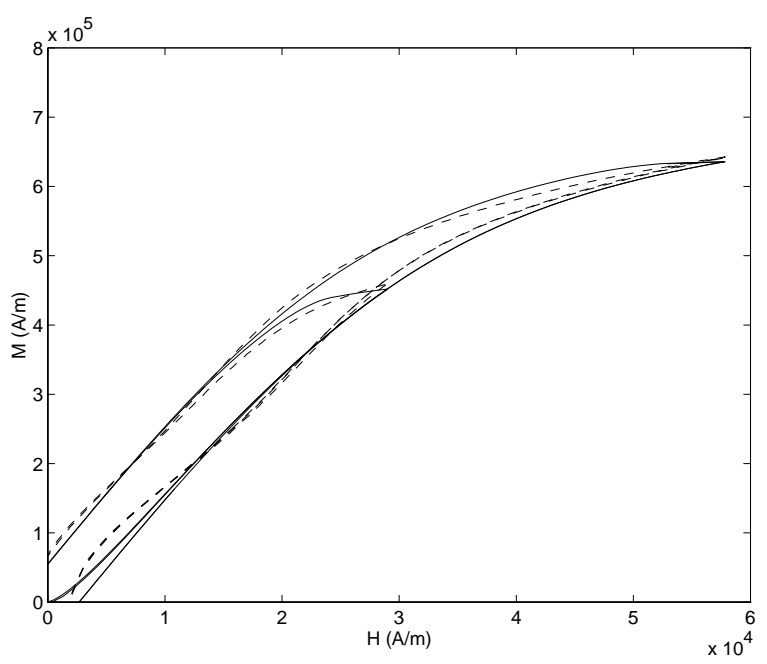

(d)

Figure 4. Experimental data (-- ) and magnetization model dynamics ( - ) for multiple drive and prestress levels; (a) Three drive levels with $1.0 \mathrm{ksi}$ applied stress, (b) Magnified view of $1.0 \mathrm{ksi}$ case, (c) Two drive levels with $1.3 \mathrm{ksi}$ applied stress, (d) Magnified view of $1.3 k$ si case. 
The model fits at three drive levels for the $1.0 \mathrm{ksi}$ case are illustrated in Figure 4a, b while fits for two drive levels with a $1.3 \mathrm{ksi}$ applied stress are illustrated in Figure $4 \mathrm{c}$, d. For each fixed prestress, the same fixed parameters in Table 2 were used to attain the model responses at the multiple drive levels. The variation in model dynamics is due solely to the changes in the input fields. This illustrates the flexibility of the model with respect to drive levels. As noted in previous discussion and summarized in Table 2, only the parameter $k$ and stress contribution $\frac{9}{2} \frac{\lambda_{s} \sigma_{0}}{\mu_{0} M_{s}^{2}}$ to $\tilde{\alpha}$ must be modified to account for changes in prestress. Hence the model is also highly flexible with respect to applied prestresses.

Close examination of Figure 4a, c indicates that one aspect of the experimental transducer behavior which is not quantified by the model is the constricted or 'wasp-waisted' behavior which occurs at low applied fields. This behavior has been noted by other investigators [18, 24] and is hypothesized to be due to $180^{\circ}$ domain changes [25]. While quantification of this effect is ultimately desired, the accuracy and flexibility of the current magnetization model are sufficient for control applications in this operating regime.

\section{Magnetostriction Model}

The second mechanism which must be modeled for the utilization of transducers in control design is the magnetostriction due to changing magnetization. Once this model is obtained, it can be combined with the previous magnetization model to provide a characterization of strains output by the transducer in terms of currents input to the solenoid. For this investigation, we considered the quadratic model (1) as a first approximation to the relation between magnetization and magnetostriction.

The performance of this model is indicated in Figure 5. At moderate drive levels, the strain data exhibits minimal hysteresis and is adequately characterized by the quadratic model. At the high drive levels illustrated in Figure 5b, the data exhibits significant hysteresis and saturates from a quadratic to nearly linear relationship as $M$ approaches its maximum value.

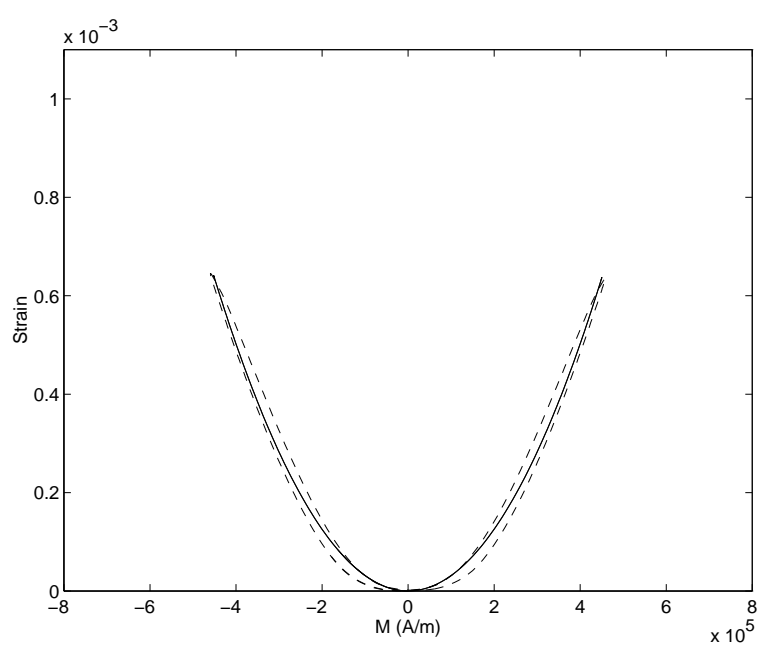

(a)

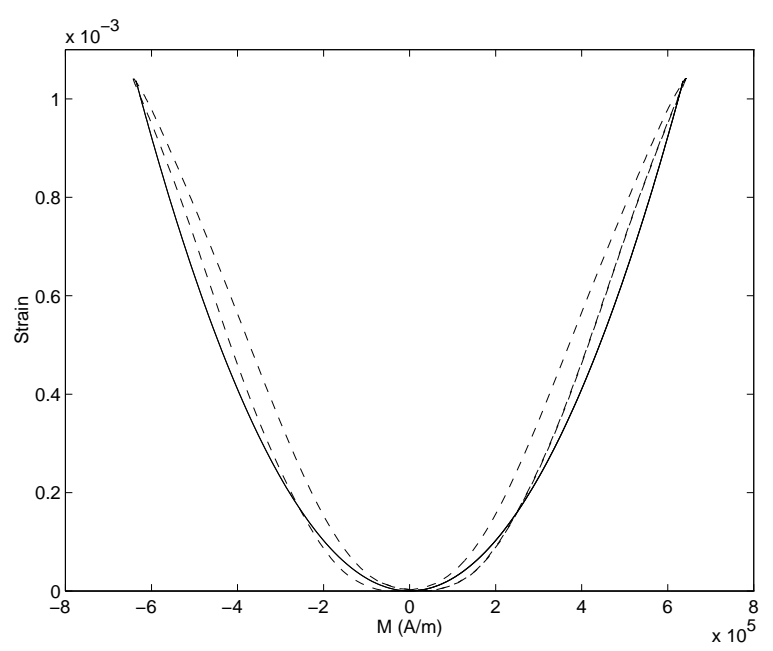

(b)

Figure 5. Experimental data (-- ) and quadratic magnetostriction model dynamics (- - ) ; (a) Low drive level, (b) High drive level. 
One component of this hysteresis is due to magnetostrictive hysteresis while other effects are due to mechanical hysteresis caused by the prestress mechanism. The performance of the model is much less accurate at high drive levels due to such unmodeled dynamics. This loss in accuracy is also reflected in the change of the estimated saturation value $\lambda_{s}=1221 \times 10^{-6}$ in the low drive regime to $\lambda_{s}=1003 \times 10^{-6}$ at the high drive level. We note that at levels below that depicted in Figure 5a, the value $\lambda_{s}=1221 \times 10^{-6}$ provides adequate model fits.

The same tendencies are apparent when the magnetization and magnetostriction models are combined to provide a relationship between input currents and output strains. As illustrated in Figure 6a, the combined model is accurate at moderate drive levels and will be adequate for control design in this regime. Figure $6 \mathrm{~b}$ illustrates that a high drive levels, however, the magnetostrictive model degenerates due to unmodeled dynamics and hysteresis. The extension of the magnetostrictive model to incorporate these effects at high drive levels is under current investigation.

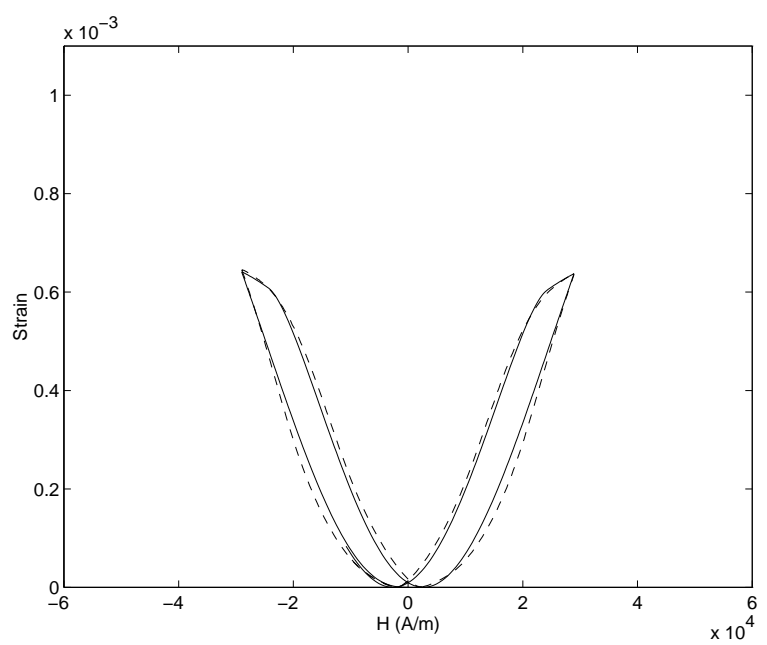

(a)

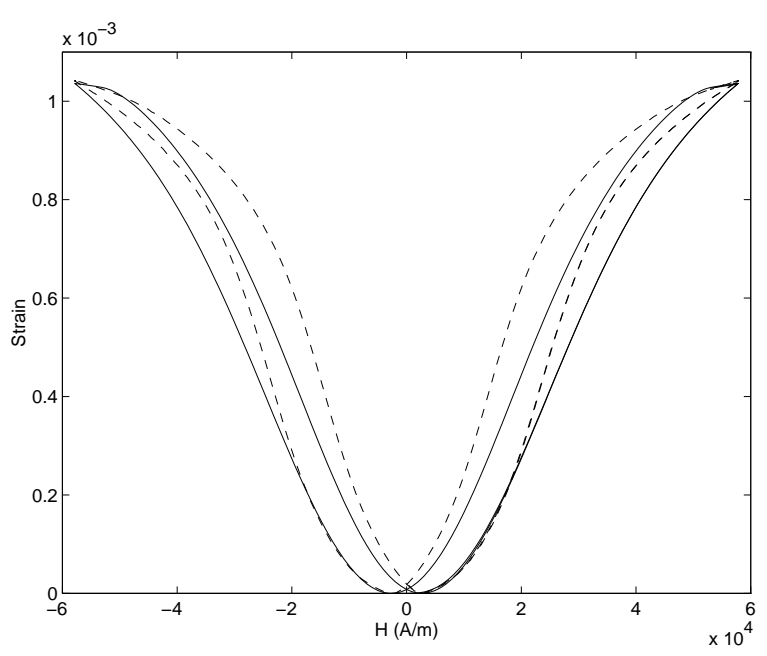

(b)

Figure 6. Experimental data $\left(--_{-}\right)$and combined magnetization and magnetostriction model dynamics (- $)$; (a) Low drive level, (b) High drive level.

\section{Minor Loop Model}

The modeling of minor asymmetric loops comprises the final component of this investigation. Accurate minor loop characterization is important for numerous applications including control design for transducers in unbiased and biased states. In a general unbiased state, it is crucial that the model be able to characterize both major and minor loop dynamics to attain the full range of dynamics specified by the control law. The characterization of minor loops in a biased state is important since it represents a common operating condition for transducers.

For both cases, we employed the volume fraction (6) to attain closure in the minor loop magnetization model. The resulting model fit is illustrated in Figure 7a, b where 1.0 ksi data containing a major loop and two minor loops is considered. Both the major and minor loop dynamics are resolved by the model with the slight discrepancy in minor loop position 
due to differences in experimental and model major loop magnetizations for the values of $H$ at which the turn points occur. We note that the model parameters for this case are those summarized in Table 2 and no parameter changes are necessary to accommodate the minor loops. As with the major loop case, turning points are dictated solely by the input magnetic field (or equivalently, the input current $I$ ). Figure $7 \mathrm{c}$ and $\mathrm{d}$ illustrate the performance of the magnetization and magnetostriction models in resolving major and minor loops in the $1.3 \mathrm{ksi}$ data. The accuracy of the fit in Figure $7 \mathrm{c}$ reflects the accuracy of the underlying magnetization model while discrepancies in the major loop strain fit in Figure $7 \mathrm{~d}$ are due to the previously mentioned unmodeled dynamics in the magnetostriction at high drive levels. At moderate levels, the minor loop model is sufficiently accurate for control applications.

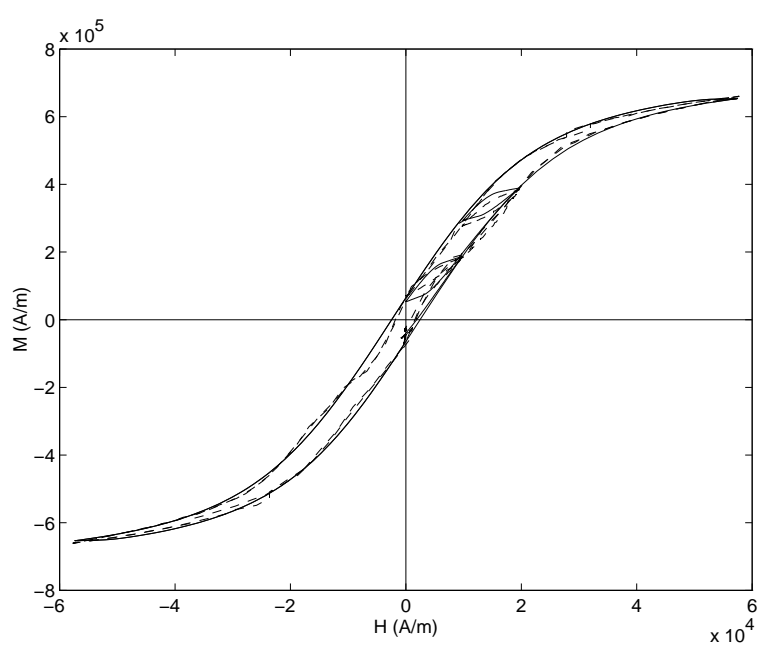

(a)

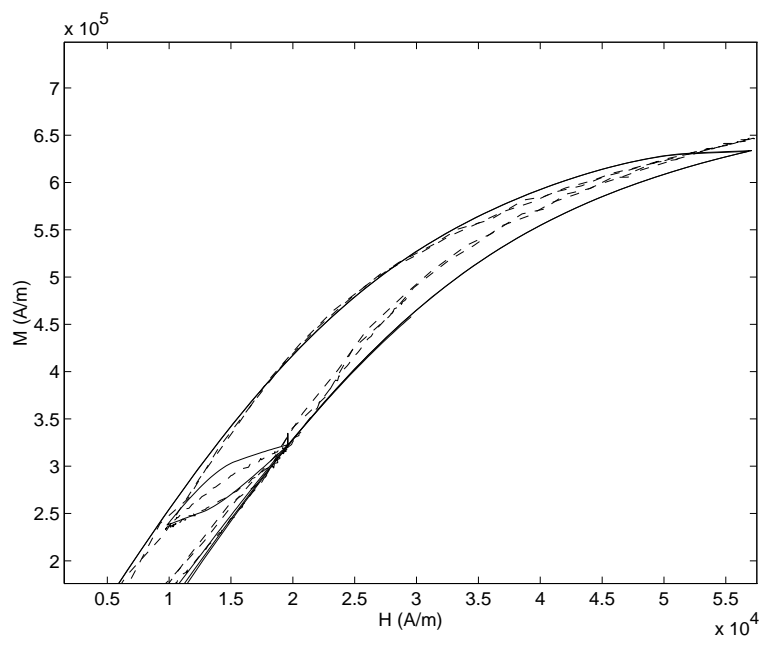

(c)

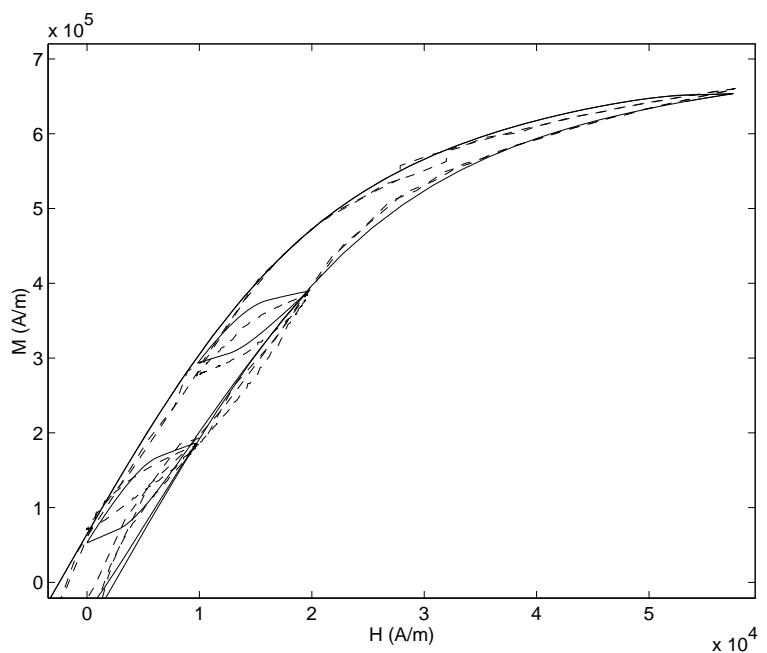

(b)

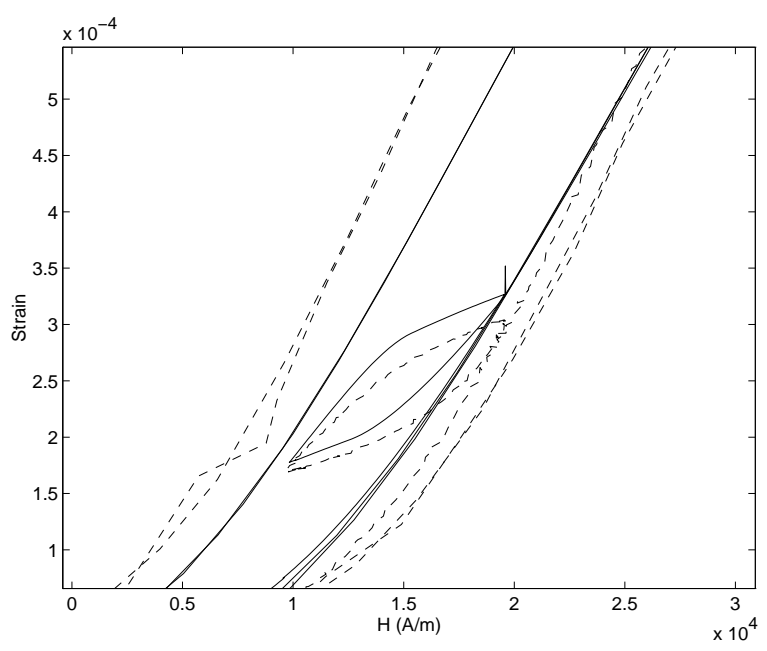

(d)

Figure 7. Experimental data (-- ) and minor loop model dynamics (- - ); (a) Two minor loops in magnetization data with $1.0 \mathrm{ksi}$ applied stress, (b) Magnified view of $1.0 \mathrm{ksi}$ case, (c) Minor loop in magnetization data with $1.3 \mathrm{ksi}$ applied stress, (d) Minor loop in strain data with $1.3 \mathrm{ksi}$ applied stress. 


\section{Concluding Remarks}

An energy-based model for characterizing magnetization and output strains for magnetostrictive transducers is presented. The magnetization model, which is based upon the JilesAtherton mean field theory for ferromagnetic materials, provides a means of characterizing the magnetic hysteresis inherent to the transducer. Through enforcement of closure conditions, nested asymmetric minor loops as well as symmetric major loops are resolved by the characterization. This magnetization model is currently constructed for a transducer with quasi-static input and fixed operating temperature. Within this regime, the model provides the capability for characterizing variable input levels to the solenoid and differing applied stresses to the Terfenol rod. The good agreement of this theory with experimental data illustrates the flexibility of the model under a variety of operating conditions.

A quadratic model based upon the geometry of moment rotations was employed to quantify the magnetostriction and strains generated by the transducer. As illustrated through comparison with experimental data, this characterization was adequate at moderate drive levels but degenerated at high drive levels due to unmodeled nonlinearities and hysteresis. Certain aspects of the magnetostriction hysteresis can be included through the energy model of [9] but adequate quantification of the full relation has not been attained and is under current investigation.

At moderate input levels, the combination of the magnetization and magnetostriction models provide and accurate characterization of output strains in terms of input currents to the solenoid. For quasi-static applications in which temperature can be regulated, the model is sufficiently accurate for control design. The robustness of the model with regard to operating conditions and the small number of required parameters (six) enhance its suitability for such applications.

\section{Acknowledgements}

The authors would like to thank Marcelo Dapino and David Jiles for numerous discussions and input regarding the modeling techniques employed here. The research of R.C.S. was supported in part by the Air Force Office of Scientific Research under the grant AFOSR F49620-95-1-0236. The research of F.T.C. and A.B.F. was supported in part by Graduate Student Research Program Grant NGT-51254, NASA Langley Research Center, Richard Silcox technical advisor, and National Science Foundation Young Investigator Award CMS 9457288.

\section{References}

[1] J.L. Butler, "Application manual for the design of ETREMA Terfenol-D magnetostrictive transducers," EDGE Technologies, Inc., Ames, IA, 1988.

[2] A.E. Clark, "Magnetostrictive rare earth-Fe 2 compounds," Chapter 7 in Ferromagnetic Materials, Volume 1, E.P. Wohlfarth, editor, North-Holland Publishing Company, Amsterdam, pp. 531-589, 1980. 
[3] F.T. Calkins, M.J. Dapino and A.B. Flatau, "Effect of prestress on the dynamic performance of a Terfenol-D transducer," Proceedings of the SPIE, Smart Structures and Integrated Systems, San Diego, CA, March 1997, Vol. 3041, pp. 293-304.

[4] M. Moffet, A. Clark, M. Wun-Fogle, J. Linberg, J. Teter and E. McLaughlin, "Characterization of Terfenol-D for magnetostrictive transducers," J. Acoust. Soc. Am., 89(3), pp. 1448-1455, 1991.

[5] E. du Trémolet de Lacheisserie, Magnetostriction: Theory and Applications of Magnetoelasticity, CRS Press, Ann Arbor, 1993.

[6] D.C. Jiles, Introduction to Magnetism and Magnetic Materials, Chapman and Hall, New York, 1991.

[7] D.C. Jiles and D.L. Atherton, "Theory of ferromagnetic hysteresis," J. Magn. Magn. Mater., 61, pp. 48-60, 1986.

[8] D.C. Jiles, J.B. Thoelke and M.K. Devine, "Numerical determination of hysteresis parameters for the modeling of magnetic properties using the theory of ferromagnetic hysteresis." IEEE Trans. Magn., 28(1), pp. 27-35, 1992.

[9] M.J. Sablik and D.C. Jiles, "Coupled magnetoelastic theory of magnetic and magnetostrictive hysteresis," IEEE Trans. Magn., 29(3), pp. 2113-2123, 1993.

[10] R.C. Smith, "Modeling techniques for magnetostrictive actuators," Proceedings of the SPIE, Smart Structures and Integrated Systems, San Diego, CA, March 1997, Vol. 3041, pp. 243-253.

[11] R.C. Smith, "Well-posedness issues concerning a magnetostrictive actuator model," Proceedings of the Conference on Control and Partial Differential Equations, CIRM, Marseille-Luminy, France, June 1997, to appear.

[12] R.C. Smith, " A nonlinear model-based control method for magnetostrictive actuators," Proceedings of the 36th IEEE Conference on Decision and Control, to appear.

[13] J. Pratt and A.B. Flatau, "Development and analysis of a self-sensing magnetostrictive actuator design," J. Intell. Mater. Syst. and Struct., 6(5). 1995, pp. 639-648.

[14] D.L. Hall and A.B. Flatau, "Nonlinearities, harmonics and trends in dynamic applications of Terfenol-D," Proceedings of the SPIE Conference on Smart Structures and Intelligent Materials, Vol. 1917, Part 2, pp. 929-939, 1993.

[15] R.C. Smith, "Hysteresis modeling in magnetostrictive materials via Preisach operators," ICASE Report 97-23; J. Math. Systems, Estimation and Control, to appear.

[16] V. Basso and G. Bertotti, "Hysteresis models for the description of domain wall motion," IEEE Trans. Magn., 32(5) pp. 4210-4213, 1996.

[17] D.A. Philips, L.R. Dupré and J.A. Melkebeek, "Comparison of Jiles and Preisach hysteresis models in magnetodynamics," IEEE Trans. Magn., 31(6), pp. 3551-3553, 1995. 
[18] B.D. Cullity, Introduction to Magnetic Materials, Addison-Wesley, Reading, MA, 1972.

[19] J.B. Thoelke, "Magnetization and magnetostriction in highly magnetostrictive materials," Master's Thesis, Iowa State University, 1993.

[20] D.C. Jiles, "Theory of the magnetomechanical effect," J. Phys. D: Appl. Phys., 28, pp. $1537-1546,1995$.

[21] D.C. Jiles and J.B. Thoelke, "Theoretical modelling of the effects of anisotropy and stress on the magnetization and magnetostriction of $\mathrm{Tb}_{0.3} \mathrm{D}_{\mathrm{y}_{0.7}} \mathrm{Fe}_{2}, "$ J. Magn. Magn. Mater., 134, pp. 143-160, 1994.

[22] D.C. Jiles, "A self consistent generalized model for the calculation of minor loop excursions in the theory of hysteresis," IEEE Trans. Magn., 28(5), pp. 2602-2604, 1992.

[23] D.L. Atherton and V. Ton, "The effects of stress on a ferromagnet on a minor hysteresis loop," IEEE Trans. Magn., 26(3), pp. 1153-1156, 1990.

[24] D.C. Jiles and S. Hariharan, "Interpretation of the magnetization mechanism in Terfenol-D using Barkhausen pulse-height analysis and irreversible magnetostriction," $J$. Appl. Phys., 67(9), pp. 5013-5015, 1990.

[25] A.E. Clark, Personal Communications. 